(Aus dem physiologischen Laboratorium der psychiatrischen und Nervenklinik zu St. Petersburg. Vorsteher: Prof. W. v. Bechterew.)

\title{
Zur Frage über die Erregbarkeit der motorischen Zentra in der Hirnrinde neugeborener Säugetiere.
}

Von

\section{Sergius Michailow.}

(Mit 23 Textfiguren.)

Das experimentelle Studium der Funktionen der Hirnrinde neugeborener Säugetiere begann bloss einige wenige Jahre nach der Veröffentlichung der bedeutungsvollen Untersuchungen Fritsch's und Hitzig's, welche gezeigt hatten ${ }^{1}$ ), dass es in der Hirnrinde bestimmte und umschriebene Partien gibt; bei deren Reizung mittelst des elektrischen Stromes Kontraktionen bestimmter, immer der gleichen Muskelgruppen eintreten.

Diese Frage wurde in bezug auf neugeborene Tiere zuerst von Soltman $n^{2}$ ) im Jahre 1876 hervorgehoben. Soltmann studierte die Frage über die Erregbarkeit der Hirnrinde an neugeborenen Hunden und Katzen, wobei die in seiner Arbeit niedergelegten Ergebnisse fast ausschliesslich an jungen Hunden erlangt sind. Es wurden von ihm zu diesem Zwecke 132 Hunde utilisiert, die vorher mit Äther, Chloroform oder Morphium narkotisiert worden waren.

Als Reiz benutzte Soltmann den konstanten Strom einer Pincus'schen Batterie, wobei die Stärke des Stromes eine derartige war, dass er von der Zungenspitze bloss leicht empfunden wurde; mitunter wurde aber der Strom bedeutend verstärkt. Soltmann

1) Fritsch und Hitzig, Über die elektrische Erregbarkeit des Grosshirns. Reichert's und Du Bois-Reymond's Archiv 1870.

2) Soltmann, Experimentelle Studien über die Funktionen des Grosshirns der Neugeborenen. Jahrb. f. Kinderheilk. u. psych. Erziehung N. F. Bd. 9. 1876. 
reizte bei neugeborenen Hunden den ganzen Lobus prae- und postfrontalis und erhielt nie irgendeinen Effekt hinsichtlich Kontraktion der Muskulatur der Extremitäten, des Gesichts, Nackens, Rückens, Bauches und Schwanzes, woraus er den Schluss zog, dass diejenigen Partien der Hirninde, die bei erwachsenen Tieren motorische Funktionen besitzen, bei Neugeborenen der gleichen Art diese Eigenschaft nicht haben. Daraufhin entstand natürlicherweise die Frage: Wann bilden sich solche motorische Rindenzentren aus? Soltm an erhielt erst am 10. Tage nach der Geburt des Tieres bei Reizung der Hirnrinde mittelst schwacher und starker Ströme Muskelzuckungen und bloss an den vorderen Extremitäten. Am 13. Tag ungefähr beginnt bei Reizung das Rindenzentrum für die Bewegung der hinteren Extremität zu funktionieren, und am 16. Tage erscheinen als schon vollkommen unıschrieben und isoliert drei Zentra: für die vorderen, die hinteren Extremitäten und die Gesichtsmuskulatur; die Zentra für die Kontraktion der Rücken- und Bauchmuskeln sowie der Muskulatur des Schwanzes bleiben noch aus. Die Rindenzentra nehmen, nach Soltmann, zuerst bedeutend grössere Partien der Hirnrinde ein; mit Vermehrung der Zahl der Zentra isoliert und umgrenzt sich jedes einzelne auf einem kleineren Gebiet, wobei es schon die dem erwachsenen Tier der gleichen Art eigene Lage einnimmit.

Ausser dieser Serie von Experimenten mit Reizung der Hirnrinde stellte Soltmann noch drei andere Serien von Experimenten an. Er exstirpierte bei Hunden, die das Alter von 9-10 Tagen noch nicht erreicht hatten, die Hirnrinde im Gebiete der motorischen Zentra und erhielt dabei nie irgendeinen Effekt im Sinne des Ausfalls der motorischen Funktionen. Dieser Effekt trat im Gegenteil ständig ein bei Tieren, die das genannte Alter überschritten hatten.

Ferner exstirpierte Soltman n die Rinde der motorischen Zone bei jungen Hunden, bei denen die Rindenzentra sich noch nicht ausgebildet hatten, und liess diese Tiere am Leben. Hierbei kamen weiterhin nie irgendwelche motorische Defekte zur Beobachtung, und soche Hunde blieben nur überhaupt in ihrer Entwicklung und ihrem Wachstum vor ihren Altersgenossen zurück. Eine vierte Reihe von Experimenten endlich, die Soltmann angestellt hatte, bestand darin, dass er mittelst des elektrischen Stromes tiefer unter der Rinde liegende Teile reizte. Auf Grund solcher Experimente kam er zu dem Schlusse, dass das Corpus striatum bei Neugeborenen 
keine motorisehen Eigenschaften besitzt, und dass Reizung der Fasern in der inneren Kapsel beständig Zuckung der gekreuzten vorderen Extremität hervorruft.

Hiermit haben wir kurz den tatsächlichen Teil der Untersuchungen Soltmann's über die Funktionen des Grosshirns neugeborener Tiere auseinandergesetzt. Solt mann selbst aber begnügte sich nicht bloss mit Tatsachen und fügte seiner Arbeit noch eine bedeutende Anzahl von Betrachtungen, Vermutungen und Voraussetzungen hinzu, welche alle darauf gerichtet waren, die an neugeborenen Hunden erlangten Tatsachen auf das Kind zu übertragen. Dabei behauptete er, dass die Ausbildung der Rindenzentra indirekter Abhängigkeit von den Eindrücken der Aussenwelt stehe, welche das neugeborene Tier mittelst seiner Sinneswerkzeuge aufnimmt. (Nach Soltmann öffnen Hunde ihre Augen am siebenten bis achten Tage nach der Geburt.) Gegen diese Verallgemeinerungen sprach sich aber schon bald darauf $\mathrm{T}$ a $\mathrm{rch}$ an off. ${ }^{1}$ ), der über die gleiche Frage - Erregbarkeit der Hirnrinde bei Neugeborenen - arbeitete, aus. Er stellte seine Experimente an Meerschweinchen an, die, wie bekannt, bei der Geburt schon äusserlich über alle dem erwachsenen Tiere eigenen Funktionen verfügen. Tarchan off benutzte Meerschweinchen im Alter von 17 Stunden resp. 3 und 5 Tagen und verglich sie mit gleichaltrigen Kaninchen. Die Reizung der Oberfläche der Hirnhemisphären geschah mittelst Elektroden von der Sekundärspirale eines du B o is-Reymond'schen Induktionsapparates, welcher von zwei Daniell'schen Elementen gespeist wurde. Auf den vorderen Partien der Hemisphären neugeborener Meerschweinchen fand Tarchan off Punkte, deren Reizung Bewegungen an den Kiefern und der vorderen und hinteren Extremität der kontralateralen Körperseite hervorrief, wobei zur Erzielung von Bewegungen in jedem einzelnen dieser Glieder der Strom entsprechend verstärkt werden muss. Auf Grund diescr Experimente zog Tarchan off.den Schluss, dass bei neugeborenen Meerscbweinchen fast von den ersten Stunden nach der Geburt an, dje, wie sie dann genannt wurden, psychomotorischen Zentra für den Kauapparat, die vorderen und hinteren Extremitäten bereits entwickelt sind. Ferner

1) Tarchanoff, Über die psychomotorischen Zentra und ihre Entwicklung beim Menschen und den Tieren. Russisch. St. Petersburg 1879. - Tarchanoff, Revue mensuelle de med. et de chir. 1878. 
wies Tarchan off nach, dass Meerschweinchen schon am Ende ihrer intrauterinen Lebensperiode wohlentwickelte psychomotorische Zentra besitzen. Bei Kaninchen dagegen, die mit geschlossenen Augen und Ohren zur Welt kommen (nach Tarchan off beginnt bei neugeborenen Kaninchen der äussere Gehörgang erst vom fünften Tage nach der Geburt an sich zu öffnen, die Augen öffnen sich vollständig am 13. Tage), beginnen die psychomotorischen Zentra vom 12. oder 13. Tage nach der Geburt an zu erscheinen, dabei entwickeln sich früher als alle übrigen die Zentra für die Bewegung des Unterkiefers und überhaupt diejenige Gruppe von Zentren, durch deren Reizung der Kauakt ausgelöst wird. Nach ihnen erscheinen die Zentra für die Vorderpfote und 3-4 Tage später die Zentra der Hinterpfote. Am 16. Tage nach der Geburt sind beim neugeborenen Kaninchen alle psychomotorischen Zentra bereits vorhanden. Nach der Meinung Tarchan off's liegt die Ursache für den höheren Entwicklungsgrad des Nervensystems resp. der psychomotorischen Zentra des neugeborenen Meerschweinchens im Vergleich zu demjenigen des neugeborenen Kaninchens nicht nur in der längeren Dauer des intrauterinen Lebens der ersteren, sondern auch in den besonders günstigen Ernährungs- und Wachstumsverhältnissen des Meerschweinchens während der intrauterinen Lebensperiode. Zur Bestätigung dieser Vermutung unternahm Tarchan off eine neue Reihe von Experimenten über den Einfluss verschiedener Bedingungen und Regime auf die Entwicklung der psychomotorischen Zentra neugeborener Kaninchen und Meersehweinchen.

Um die Ernährung des Gehirns zu steigern, hielt er die Versucbstiere täglich während $3 / 4-2$ Stunden nacheinander in senkrechter Lage mit dem Kopf nach unten und verabreichte ihnen innerlich Phosphor in der Dosis von 1/80 Gran, in Lebertran gelöst - ein oder zweimal täglich. Zur Erzielung des entgegengesetzten Effektes führte er dem Tier täglich 2-4 Teelöffel einer $35 \%$ igen Alkohollösung ein und brachte es ebenfalls täglich für $3 / 4-2$ Stunden in eine-senkrechte Lage mit dem Kopf nach oben und den Beinen nach unten. Auf Grund solcher Experimente kam Tarchan off zu dem Schluss, dass einerseits Phosphor und die Hyperämie des Gehirns, andererseits der Alkohol und die Hirnanämie in der Tat einige von denjenigen Bedingungen sind, welche die Entwicklung der psychomotorischen Zentren und überhaupt des Nervensystems beeinflussen (die ersteren beschleunigen diese Entwicklung - die letzteren ver- 
langsamen sie). Auf Grund dieser kurz von uns angeführten Untersuchungen spricht sich Tarchan off dahin aus, dass auf solche Weise bei manchen Tierarten sich schon während der intrauterinen Lebensperiode in der Hirnrinde psychomotorische Zentren ausbilden, ganz unabhängig von den Einflüssen der Aussenwelt, kraft bloss der Ernährungs- und Wachstumsprozesse der Gewebe. Diese Tatsache zeigt, dass die Schlussfolgerung Soltmann's von dem Fehlen psychomotorischer Zentren bei neugeborenen Tieren nur auf solche beschränkt werden muss, die blind zur Welt kommen und zu einer regelmässigen Lokomotion nicht fähig sind; andererseits erscheint der Entwicklungsprozess dieser Zentren selbst, der nach Soltmann gewissermaassen nur ein Produkt der Einwirkung der Aussenwelt auf Sinnesapparate des Neugeborenen ist, tatsächlich gar nicht als solches. Und abgesehen davon, dass schon Tarchan off die Schlussfolgerungen Soltmann's beschränkt und eingeengt hat, bestritten in den nächstfolgenden Jahren viele Autoren die Richtigkeit selbst der faktischen Seite der Soltmann'schen Mitteilungen. (Lemoine, Marcacei u. a.) Lemoin ${ }^{1}$ ) war der erste, der sich dahin aussprach, dass die Hirnrinde neugeborener Hunde und Katzen im Gebiete der Gyri sigmoidei in einem bedeutend früheren Alter also das von Soltmann angegebene erregbar erscheint. Marcacci ${ }^{2}$ ) erhielt die gleichen Resultate bei der Untersuchung von sechs Hunden, die noch vor Ende der Schwangersehaft aus dem Mutterleibe entfernt worden waren, und auch von zwei Hunden und zwei Katzen, die das Alter von 1-2 Tagen erreicht hatten. Er benutzte dabei die Chloroformnarkose und erzielte Bewegungen in den vorderen, mitunter auch den hinteren Extremitäten der gekreuzten Seite. Bei Verstärkung des elektrischen Stromes kamen aber die Extremitäten beider Körperhälften in Aktion. Alle die Bewegungen erhielt Marcacci nur in dem Falle, wenn die Elektroden ins Hirngewebe in der Nähe des Sulc. cruciatus leicht versenkt wurden; bei Reizung durch blosses Anlegen der Elektroden an die Hirnoberfläche blieb in seinen Experimenten der Effekt aus. Aus diesen Tatsachen zog Marcacei den Schluss, dass die Rindenschicht der grauen Mark-

1) Lemoine, Contribution à la détermination et à l'étude expérimentale des localisations fontionelles encéphaliques. Thèse de Paris 1880.

2) Marcacci, Étude critique expérimentale sur les centres moteur corticaux. Arch. ital. de Biol, t. 1. 1882. 
substanz nicht erregbar ist, und dass das Gehirn neugeborener Tiere und selbst solcher, die sich noch im Mutterleib befinden, in der gleichen Weise wie das Gehirn erwachsener Tiere reagiert. In einer anderen Arbeit weist aber Marcacei ${ }^{1}$ ) darauf hin, dass wenn man zur Erzielung eines Effektes bei Reizung der Hirnrinde frühgeborener Tiere auch in der Tat die Elektroden 1-2 $\mathrm{mm}$ tief in die Hirnrinde versenken muss, er bei Tieren, die das Alter von 2 Tagen erreicht hatten, charakteristische Zuckungen der gekreuzten Extremitäten durch blosses Anlegen der Elektroden an die Hirnoberfläche hervorrufen konnte (Appoggiando leggiermente). Gegen diese Behauptungen äusserte sich Crosnier de Varigny ${ }^{2}$ ) aus dem Vulpian'scheu Laboratorium. Er konnte sich nicht von der Erregbarkeit der Hirn. rinde durch den elektrischen Strom an zwei Hunden, die das Alter von 1-2 Tagen erreicht hatten, weder bei Anwendung der Chloralhydratnarkose noch im wachen Zustande überzeugen.

Paneth ${ }^{3}$ ) war der Meinung, dass das Misslingen der Versuche Soltmann's, Crosnierde Varigny's und zum Teil Marcacei's hanptsächlich durch die Anwendung der Narkose bedingt sei und führte deshalb seine Untersuchungen stets ohne Narkose aus. Paneth hat seine Experimente an zwei Hunden im Alter von 18 Stunden, einem im Alter von 24, zwei im Alter von 36 und vier im Alter von 48 Stunden angestellt. Bei vier von diesen Hunden experimentierte er dabei an beiden Hemisphären und rechnete deshalb diese Experimente doppelt. Er erhielt ein positives Resultat in acht Experimenten, ein wahrscheinliches in vier und ein negatives in einem. Paneth kam zu dem Schlusse, dass die Hirnrinde junger Hunde schon in den ersten Tagen des extrauterinen Lebens erregbar sei, wobei diese erregbare Region ein Gebiet von $1-2 \mathrm{~cm}$ in der Umgebung des Sulcus cruciatus einnehme, während der übrige Teil der Hirnoberfläche unerregbar bleibe.

Nach den ursprünglichen Angaben von Bechtere ${ }^{4}$ ) geschieht

1) Marcacci, Zentri motori corticali. Estrato dal giornale della R. Academia di Torino. Torino 1882.

2) Crosnier de Varigny, Recherches expérimentales sur l'excitabilité de circonvolutions cérébrales. Paris 1884.

3) Paneth, Über die Erregbarkeit der Hirnrinde neugeborener Hunde. Arch. f. d. ges. Physiol. Bd. 37. 1885.

4) v. Bechterew, Über die Erregbarkeit der motorischen Rindenzentren bei neugeborenen Hunden. Wratsch 1886. Russisch. 
die Entwicklung der motorischen Rindenzentren beim Hunde lange nicht zu den gleichen Terminen; bei den einen treten die ersten Spuren der Erregbarkeit der Zentren schon am 10. Tage auf, bei den anderen sind sie noch bis zum 12. bis 14., ja sogar 15. Tag noch unerregbar. Ausserdem gab v. Bechterew an, dass gar keine Beziehungen zwischen dem Öffnen der Augen und der Entwicklung der genannten Zentren existieren, worauf Soltmann hinwies; bei Katzen sind die motorischen Zentren schon nach 2-3 Tagen nach der Geburt erregbar, wäbrend sie die Augen erst 7-8 Tage nach der Geburt öffnen. Früher als die übrigen entwickeln sich die Zentren der Extremitäten, ferner diejenigen für die Bewegung der Obren und des Gesichts und später als die anderen die Zentren für die Nacken- und Rückenmuskulatur und die Muskulatur des Schwanzes. Gleich von! vornherein lokalisieren sich die Zentren fast ausschliesslich im Bereich des Gyrus sigmoideus, wobei sie beinah die gleiche Lage wie beim erwachsenen Tiere einnehmen: das Zentrum für die hintere Extremität lokalisiert sich im hinteren Abteil des Gyrus sigmoideus in der Nähe von der sagittalen Hirnspalte; das Zentrum für die vordere Extremität - im vorderen Teil der gleichen Windung, in der Nachbarschaft vom äusseren Rande des Sulcus cruciatus, und etwas nach aussen und hinten von diesem Zentrum findet sich das Zentrum für die Gesichtsmuskulatur. Nach der Ansicht von Bechterew besteht der Unterschied zwischen dem erwachsenen und dem neugeborenen Tiere in bezug auf die Lokalisation der motorischen Rindenzentren darin, dass es beim erwachsenen Tiere eine grössere Zahl solcher Zentren gibt und die Reizung jedes einzelnen von ihnen die Zuckung nur einer bestimmten Gruppe von Muskeln des betreffenden Körpergliedes hervorruft, wahrend es beim neugeborenen Tiere nicht mehr als drei erregbare Punkte gibt und die Reizung jedes einzelnen eine allgemeine Zuckung der Muskulatur des betreffenden Körperabschnitts hervorruft (z. B. der vorderen oder hinteren Extremität oder des Gesichts). Nur allmählich differenzieren sich diese Zentren, ihre Lokalisation beibehaltend in der Weise weiter, dass an Stelle eines allgemeinen Zentrums für ein bestimmtes Körperglied mehrere Punkte erscheinen. Bechterew berücksichtigte in der angefübrten Arbeit noch folgende vier Punkte. Er wies darauf hin, dass die motorischen Zentren des Neugeborenen sich durch eine auffallende Erschöpfbarkeit auszeichnen, wobei, je jünger das Tier, desto schneller tritt die Erschöpfung und desto länger keine Wieder- 
herstellung der Erregbarkeit ein. Ferner gab er an, dass es nie gelingt, von der Hirnrinde neugeborener Hunde klonische Krampfbewegungen eines Körpergliedes oder einen epileptischen Anfall hervorzurufen. Die ersten Spuren von Erregbarkeit der motorischen Zone in der Hirnrinde neugeborener Tiere stehen zu der Entwicklung der Riesenzellen der Hirnrinde, als zum Auftreten von Myelin in den Fasern des Pyramidenstranges in Beziehung. Und endlich: entsprechend dem, wie die Entwicklung der motorischen Rindenzentren vorwärts schreitet, erscheinen immer schwächere Stösse als genügend, um, als Reizmittel angewandt, den gleichen motorischen Effekt zu erzielen, welcher Umstand von der Verdickung der Myelinscheide in den Fasern des Pyramidenstranges abhängt.

Langlois ${ }^{1}$ ) stellte seine Untersuchungen über die motorischen Zentren Neugeborener an Hunden, Katzen und Meerschweinchen an, wobei er mit den zwei ersten Tierarten Resultate erhielt, welche sich mit denjenigen Soltmann's und v. Bechterew's decken. Die Meersehweinchen, die ihm zur Untersuchung dienten, waren 15 Stunden bis 2 Tage alt, wobei Langlois stets Narkose, und zwar Chloroform-, Äther- oder öfter Morphiumnarkose anwandte. Im letzteren Falle bekamen die jungen Meerschweinchen mit einem Körpergewicht von 150-200 g 4-6 cem Morphii hydrochlorici. Durch Reizung eines Punktes, der $4-5 \mathrm{~mm}$ vom Ende der Kreuzfurche entfernt liegt, wurde bei solchen Tieren der Kauakt ausgelöst. Reizung des Rindengebietes vor und hinter dieser Furche und in der Nähe der sagittalen Hirnspalte erwies sich bei der gleichen Stromstärke als unwirksam und musste nach Langlois zur Erzeugung eines motorischen Effektes von diesem Gebiete aus der Strom verstärkt werden. Hierbei tritt eine Bewegung der Extremitäten ein, wobei die vordere Extremität bei schwächeren Strömen in Bewegung gerät als die hintere. Die exaktesten und beständigsten Resultate erhielt Langlois an Meerschweinchen, die das Alter von 2 Tagen erreicht hatten.

In demselben Jahre veröffentlichte Bechterew ${ }^{2}$ ) wiederum eine Arbeit über die Erregbarkeit verschiedener Gehirnteile bei neu-

1) Langlais, Notes sur les centres psychomoteurs des nouveau-nés. Compt. rend. de la Société de Biol. Paris 1889.

2) v. Bechterew, Über die Erregbarkeit verschiedener Hirnteile neugeborener Tiere. Wratsch 1889. Russisch. 
geborenen Tieren, in welcher er hinsichtlich der Erregbarkeit der motorischen Rindenzentren wiederholt, dass bei neugeborenen Hunden die Erregbarkeit dieser Zentren zuerst im Alter von 10-13 Tagen nach der Geburt auftritt. Nach der Ansicht von Bechterew lässt sich bei neugeborenen ebenso wie bei erwachsenen Tieren eine Einteilung der motorischen Punkte in leicht- und in schwererregbare durchführen. Während bei neugeborenen Iunden bei Reizung der leicht erregbaren im Gebiet des Gyrus sigmoideus liegenden motorischen Punkte schon Bewegungen in den Extremitäten auftreten, erscheinen die schwer erregbaren nach hinten vom Gyrus sigmoideus liegenden motorischen Punkte (z. B. die Punkte für die Bewegung der Ohren und Augen) als noch völlig unerregbar, und erst viel später beginnt ihre Reizung die entsprechenden Bewegungen hervorzurufen. So gelang es Bechterew erst eine Woche nach dem ersten Auftreten von Bewegungen in den Extremitäten durch Reizung des Gyrus sigmoideus eine Bewegung in dem Schwanze und den Ohren hervorgerufen, und konjugierte Bewegung der Augäpfel bei Reizung der Oberfläche des Lobus occipitalis kam nicht vor Ende des ersten Monats zum Vorsehein.

In der dritten seiner die erwähnte Frage behandelnden Arbeiten kam Bechterew bei Berücksichtigung 1. dessen, dass seine neuen zusarnmen mit Bary angestellten Experimente gezeigt hatten, dass es in einzelnen Fällen gelingt, durch Reizung der Hirnrinde Zuckungen in den Extremitäten selbst bei 24 Stunden alten Hunden zu erzeugen, und 2. dessen, dass es in $1 / 3$ aller an neugeborenen Hunden ausgeführten Experimenten unmöglich war, bei Reizung der Hirnrinde Bewegungen zu erzielen, die bei den meisten anderen Tieren des gleichen Alters auslösbar sind, zu der Überzeugung, dass die Erregbarkeit der Hirnrinde neugeborener Tiere von ganz verschiedenen und nicht selten zufälligen Bedingungen abhängt, unter welchen neben der grösseren oder geringeren Reife des neugeborenen Tieres wahrscheinlich auch individuelle Abweichungen in Betracht zu : ziehen sind. In dieser Unbeständigkeit der Resultate, die bei Reizung der Hirnrinde Neugeborener der gleichen Art erzielt werden, sieht Bechterew eine genügende Erklärung für die Widersprüche in den Ansichten der Autoren hinsichtlich der Zeit des ersten Auf-

1) v. Bechterew, Über die Erregbarkeit der Hirnrinde neugeborener Tiere. Obosrenije Psychiatrii 1897. Russisch. 
tretens von Erregbarkeit in den motorischen Rindenzentren neugeborener Tiere.

Bary ${ }^{1}$ ) wandte bei seinen Untersuchungen nie die Narkose an. Die Experimente wurden an neugeborenen Hunden und Katzen im Alter von einigen Stunden bis zu einem Monat und mehr angestellt. Ausserdem wurden einige Experimente an neugeborenen Kaninchen und Meersehweinchen ausgeführt. Auf Grund dieser Experimente kam Bary zu folgenden Schlüssen: bei neugeborenen Hunden und Katzen gelingt es durch Reizung mittelst eines äusserst schwachen faradischen Stromes bestimmter in Nachbarschaft des Sulcus cruciatus liegender Punkte der Hirnrinde Bewegungen der Extremitäten hervorzurufen; bei dem anderen Teil der gleichalterigen Tiere lassen sich durch Reizung der Hirnrinde mittelst Strömen von derselben Stärke Bewegungen nicht auslösen; es fehlt die einzelne Bewegung in den verschiedenen Gelenken, die Extremität kontrahiert sich aber auf einmal in allen Gelenken; die Zentren erschöpfen sich äusserst leicht; vom fünften bis achten Tage an gelingt es gleichzeitig mit einer allgemeinen Kontraktion der Extremität auch Bewegung in den Gelenken hervorzurufen; eine wesentliche Rolle spielt bei der Entwicklung der Rindenzentren die Individualität; zuerst bei einem mindestens 2 Monate alten Tiere gelingt es, durch Faradisation der Hirnrinde einen epileptischen Anfall auszulösen.

Endlich berührt Bechterew ${ }^{2}$ ) in seinem kapitalen Werke der letzten Jahre wieder die Frage über die Erregbarkeit der Rindenzentren neugeborener Tiere und kommt sowohl auf Grund seiner eigenen Untersuchungen über diese Frage als auch auf Grund der Untersuchungen anderer Forscher zum Schlusse, dass bei neugeborenen Tieren die motorischen Rindenzentren nicht vollkommen entwickelt sind.

Von dem Umstande ausgehend, dass, wie gezeigt, über die im Titel zu vorliegender Arbeit angegebene Frage unter den Autoren eine grosse Meinungsverschiedenheit herrscht, erschien es als nicht überflüssig, nochmals eine Untersuchung der Erregbarkeit der moorischen Rindenzentren neugeborener Tiere vorzunehmen. Ich er-

1) Bary, Über die Erregbarkeit der Hirnrinde neugeborener Tiere. Dissert. St. Petersburg 1898. Russisch.

2) v. Bechterew, Grundzüge der Lehre von den Hirnfunktionen. Teil VI. St. Petersburg 1906. Russisch. 
griff deshalb den Vorschlag meines hochgeehrten und teuren Lehrers, Akademiker Bechterew, mich mit dieser Frage zu beschäftigen, wobei er sehon damals betonte, dass einige der genannten Zentren bei neugeborenen Tieren überhaupt noch nicht gefunden worden seien (z. B. die Rindenzentren für die Kontraktion der Pupille usw.).

Als Untersuchungsobjekt dienten fast ausschliesslich neugeborene Hunde, in den ersten Tagen nach der Geburt und einige Meerschweinchen. Die Operation der Eröffnung des Schädels und Blosslegung der Rindenoberfläche der Hirnhemisphären wurde auf die übliche Weise ausgeführt, wobei die drei folgenden Bedingungen stets angestrebt wurden: 1. Schnelligkeit der Operation, 2. Abheben der ganzen Schädeldecke womöglich in einer Etappe, jedenfalls in möglichst grossen Stücken, 3. Schonen des Blutsinus der harten Hirnhaut. Diese drei Ziele wurden stets angestrebt und in fast allen Fällen (ausser dem ersten, provisorischen) erreicht, und hierdurch wurden vermieden: 1 . Eintritt einer Chokwirkung durch Schmerz, da bei den Untersuchungen Narkose nicht angewandt wurde, 2. Bildung einer Hirnhernie, 3. bedeutende Blutverluste usw. Ich will hier nicht auf diejenigen Vorteile hinweisen, welche mit der Beseitigung all dieser schädlichen Momente verbunden sind, weil die Angaben von v. Bechterew, Paneth, Bary u. a. deutlich gezeigt haben, dass diese Momente jedes Experimentieren unmöglich machen, da sie das Experiment zu keinen Resultaten kommen lassen.

Ausserdem war schon seit lange der schädliche Einfluss der Abkühlung des Tieres und der entblössten Hirnrinde auf die Erregbarkeit ihrer Zentren festgestellt; deshalb wurden unsere Tiere während der ganzen Dauer des Experimentes in Watte eingehullt gehalten, und wurde die Hirnoberfläche fast ununterbrochen bald mit physiologischer Kochsalzlösung (von der $\mathrm{t}^{0}=38^{\circ} \mathrm{C}$.), bald, was eigentlich fast immer ausgeführt wurde, mit Ringer-Lockescher Flüssigkeit, welche bis zur angegebenen Temperatur erwärmt war, und die von $\mathrm{Locke}^{1}$ ) ursprünglich angegebene Zusammensetzung:

(KCl) Kalium chloratum . . . 0,02\%,

$\left(\mathrm{NaHCO}_{3}\right)$ Natrium bicarbonicum $0,02 \%$,

$\left(\mathrm{CaCl}_{2}\right)$ Calcium chloratum . - $0,02 \%$,

$\left(\mathrm{C}_{6} \mathrm{H}_{12} \mathrm{O}_{6}\right)$ Saccharum uricum . . 0,10\%,

( $\mathrm{NaCl})$ Natrium chloratum . . 0,90\%, hatte, berieselt.

1) Locke, Zentralbl. f. Physiol. Bd. 14. 
Zur Reizung der Hirnrinde wurde der elektrische Strom von einem Akkumulator, der mit einem du Bois - Re y mond'schen Schlittenapparat und dessen Vermittlung mit zwei Platinelektroden in Verbindung stand, benutzt. Der Rollenabstand variierte etwas in den verschiedenen Experimenten; genaue diesbezügliche Angaben werden in den Protokollen der einzelnen Experimente angeführt sein.

Experiment am 27. Juni 1908. Nr. 1.

Junger Hund, geboren am 27. Juni 1908. Zur Zeit des Fxperimentes war er ungefähr 12 Stunden alt. Körpergewicht: $450 \mathrm{~g}$;

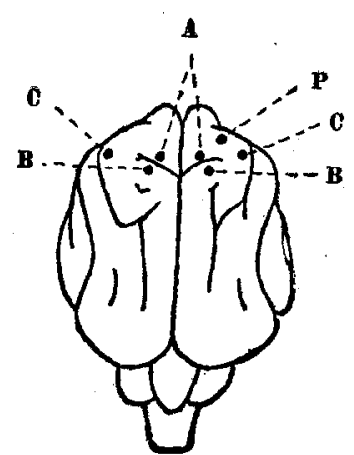

Fig. 1.

Körperlänge: $16 \mathrm{~cm}$. Reizung der motorischen Rindenregion führte bei einem Rollenabstand von $18-15-12 \mathrm{~cm}$ zu keinem motorisehen Effekt; bei einem Rollenabstand von $10 \mathrm{~cm}$ trat ein:

1. Kontraktion der Nackenmuskulatur mit Zurückwerfen des Kopfes nach oben und hinteu bei Reizung des Punktes $P$ (Fig. 1);

2. Zuckung (allgemeine) der gekreuzten Vorderpfote bei Reizung des Punktes $A$ (Fig. 1);

3. Zuckung (allgemeine) der gekreuzten Hinterpfote bei Reizung des Punktes $B$ (Fig. 1);

4. Öffnen und Schliessen des Maules, đ. h. ein Kauakt, bei Reizung des Punktes $C$ (Fig. 1).

\section{Experiment am 5. Juli 1908. Nr. 2.}

Junger Hund, geboren am 27. Juni 1908. Körpergewicht: $580 \mathrm{~g}$; Körperlänge: $25 \mathrm{~cm}$. Reizung der motorischen Rindenregion blieb bei einem Rollenabstand von 18-16-15 cm ganz ohne Effekt; auf Reizung bei einem Rollerabstand von $14 \mathrm{~cm}$ trat ein:

1. Anziehen und Aufbeben der gekreuzten Hinterpfote bei Reizung des Punktes $B$ (Fig. 2);

2. Bewegung des Schwanzes bei Reizung des Punktes $Q$ (Fig. 2);

3. Auftreten und Kontráktion vornehmlich im Kubitalgelenk der gekreuzten Vorderextremität bei Reizung des Punktes A (Fig. 2);

4. Drehung des Kopfes um eine senkrechte Achse (d. b. Seitwärtsbewegung des Kopfes) nach der entgegengesetzten Seite bei Reizung des Punktes $F$ (Fig. 3);

5. Wendung des Kopfes um die sagittale Körperachse (d. h. Drehbewegung des Kopfes), wobei das Frontalgebiet sich nach der 
Seite der Reizung, das Okzipitalgebiet in entgegengesetzter Rïchtung bei Reizung des Punktes $G$ (Fig. 3) bewegt;

6. konjugierte Bewegung der Augäpfel bei Reizung des Punktes $\boldsymbol{E}$ (Fig. 2) auf der rechten Hemisphäre, wobei das linke Auge nach

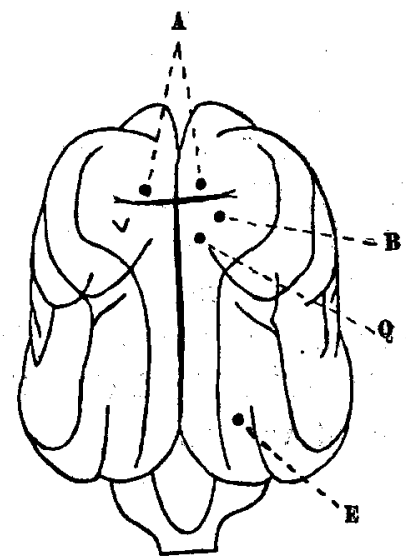

Fig. 2. oben aussen, das rechte nach oben innen gerichtet war. Die Augenlider waren abgeschnitten.

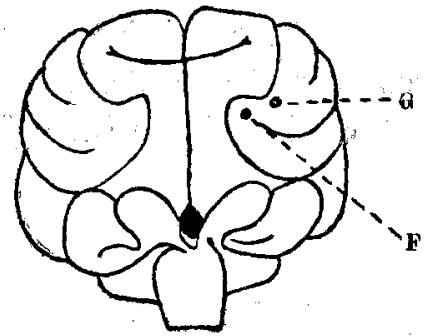

Fig. 3.

Experiment am \%. Juli 1908. Nr. 3.

Junger Hund, geboren am 27. Juni 1908. Körpergewicht $640 \mathrm{~g}$, Körperlänge: $28 \mathrm{~cm}$. Reizung der motorischen Rindenzentren löste bei einem Rollenabstand von $14 \mathrm{~cm}$ aus :

1. Bewegung der Augäpfel (konjugierte) bei Reizung des Punktes $E$ (Fig. 4) an der linken Hemisphäre, wobei das rechte Auge nach oben aussen, das linke nach oben innen gerichtet war

2. Verbreiterung der Pupille bei Reizung des Punktes $D$ (Fig. 4);

3. Anziehen und Aufheben der gekreuzten Hinterpfote bei Reizung des Punktes $B$ (Fig. 4);

4. Ausstrecken und Geraderecken der gekreuzten Hinterpfote bei Reizung des Punktes $\boldsymbol{H}$ (Fig. 4);

5. Bewegung (vornehmlich Einkneifen) des Schwanzes bei Reizung des Punktes $Q$ (Fig. 4);

6. Schliessen der Augenlider bei Reizung des Punktes $K$ (Fig. 4);

7. Bewegung der Oberlippe bei Reizung des Punktes $M$ (Fig. 4);

8. Verbreiterung der Pupille bei Reizung des Punktes $N$ (Fig. 4);

9. konjugierte Bewegung der Augäpfel bei Reizung des Punktes $U$ (Fig. 5) an der rechten Hemisphäre, wobei "das linke "Auge nach aussen unten, das rechte nach innen unten gerichtet war; 
10. Wendung des Kopfes um eine senkrechte Achse (d. h. Seitwärtsbewegung des Kopfes) nach der entgegengesetzten Seite bei Reizung des Punktes $F$ (Fig. 5);

11. Wendung des Kopfes um die sagittale Körperachse (d. h. Drehbewegung des Kopfes), wobei sich das Frontalgebiet nach der Seite der Reizung, das 0ccipitalgebiet in entgegengesetzter Richtung bei Reizung des Punktes $G$ (Fig. 5) bewegt;

12. Biegen des Rumpfes mit der Konkavität nach der entgegengesetzten Seite bei Reizung des Punktes $R$ (Fig. 4).

Wiederholte Reizung des motorischen Rindengebietes bei einem Ineinanderschieben der Rollen auf den Abstand von 10-5-3-21-0 cm löste keine Krämpfe aus. Vor Eröffnung des Schädels sind

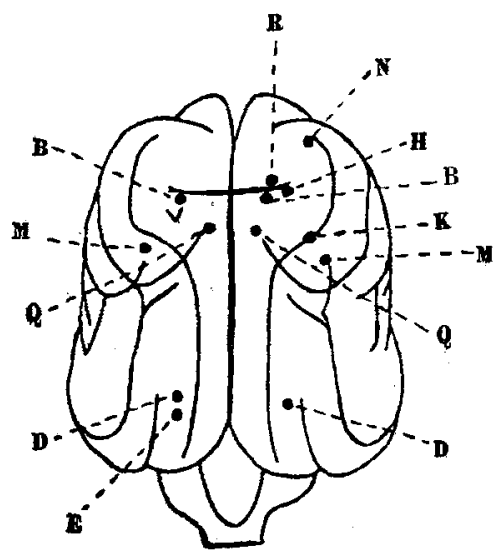

Fig. 4. die Angenlider durch einen Schnitt getrennt worden.

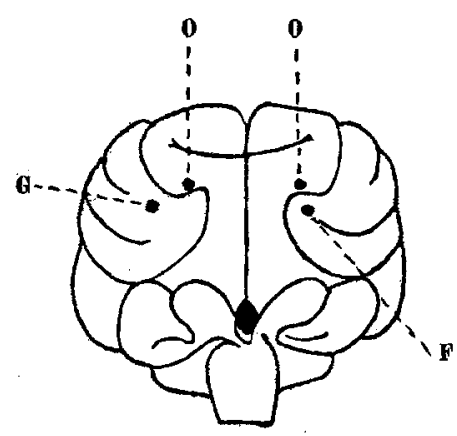

Fig. 5.

Experiment am 31. Juli 1908. Nr. 4.

Junger Hund, geboren am 28. Juli 1908. Körpergewicht: $500 \mathrm{~g}$; Körperlänge: $16 \mathrm{~cm}$. Reizung der motorischen Rindenregion bei einem Bollenabstand von 18-15-12 em blieb ohne jeden Effekt in der Bewegungssphäre.

Reizung bei einem Rollenabstand von $10 \mathrm{~cm}$ gab:

1. energische Kontraktion der gelsreuzten Vorderextremität bei Reizung des Punktes $A$ (Fig. 6);

2. Kontraktion der gekreuzten Hinterextremität bei Reizung des Punktes $\boldsymbol{B}$ (Fig. 6);

3. Bewegung der Augäpfel nach der der Reizung entgegengesetzten Seite und etwas nach unten bei Reizung des Punktes $E$ (Fig. 6). Die Augenlider sind durch Schnitt getrennt worden; 
Zur Frage über die Erregbarkeit der mot. Zentra in der Hirnzinde etc. 59

4. Kontraktion der Nackenmuskulatur mit Zurückwerfen des Kopfes nach oben und hinten bei Reizung des Punktes $P$ (Fig. 6). Bei Verstärkung des Stromes bis zu einem vollständigen Ineinanderschieben der Rollen gelang es nicht, einen epileptischen Anfall hervorzurufen.

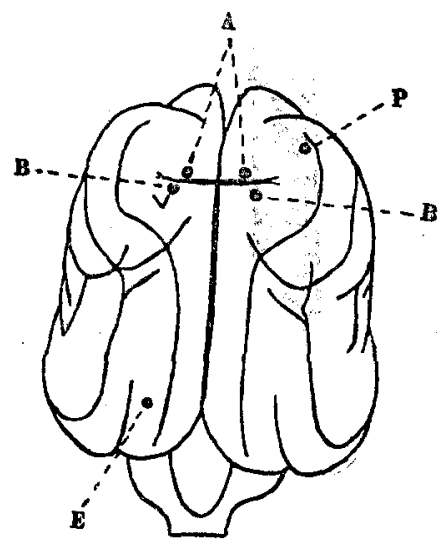

Fig. 6.

Experiment am 2. August 1908. Nr. 5.

Junger Hund, geboren am 28. Juli 1908. Körpergewicht: $550 \mathrm{~g}$; Körperlänge: $18 \mathrm{~cm}$. Reizung der Hirnrinde gab bei einem Rollenabštand von $12 \mathrm{~cm}$ :

1. Biegen im Kubitalgelenk und ferner Wälzen unter sich, der

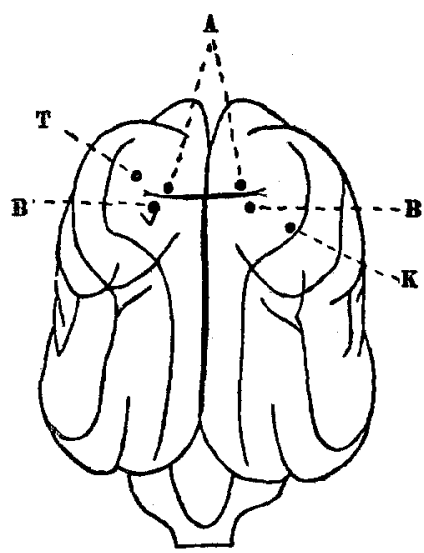

Fig. 7. gekreuzten Vorderextremität bei Reizung des Gebietes um den Punkt $A$ (Fig. 7) herum;

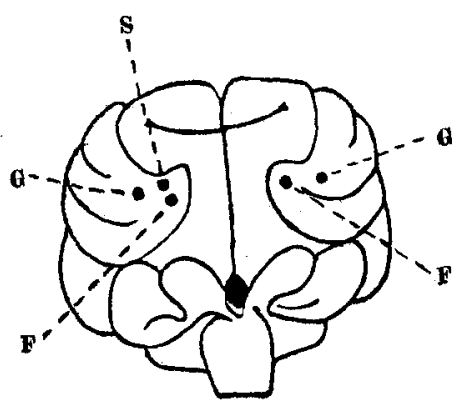

Fig. 8.

2. Bewegung des Kopfes um die sagittale Körperachse bei Reizung des Punktes $S$ (Fig. 8) an der rechten Hemisphäre, wobei 
das Frontalgebiet sich nach dér entgégengesetzten linken, das Occipitalgebiet nach der Seite der Reizung bewegt (Rollbewegung des Kopfes);

3. Aufrichten des Obres bei Reizung des Punkes $T$ (Fig. 7);

4. Einziehen des Augapfels und ein gewisses Zucken in den basalen Abschnitten der Augenlider, welche in diesem Experiment zwecks besserer Besichtigung der Augen abgeschnitten worden waren, bei Reizung des Punktes $\boldsymbol{K}$ (Fig. 7 );

5. Abduktion und Extension (Geradestrecken) der gekreuzten Vorderextremität mit Extension der Zehen bei Reizung neben dem Punkte $A$ (Fig. 7);

6. Biegen vornehmlich im Kniegelenk der gekreuzten Hinterextremität bei Reizung des Punktes B (Fig. 7);

7. Drehung des Kopfes um die senkrechte Achse nach der entgegengesetzten Seite bei Reizung des Punktes F (Fig. 8);

8. Drehung des Kopfes um die sagittale Körperachse des Punktes $G$ (Fig. 8), wobei sich das Frontalgebiet nach der Seite der Reizung, das Occipalgebiet in entgegengesetzter Richtung bewegt.

\section{'Experiment am 4. Augnst 1908. Nr. 6.}

Junger Hund, geboren am 28. August 1908: Körpergewicht: $725 \mathrm{~g}$; Körperlänge: $23 \mathrm{~cm}$. Nach Eröffnung :der Sèhädelhöhle èr-

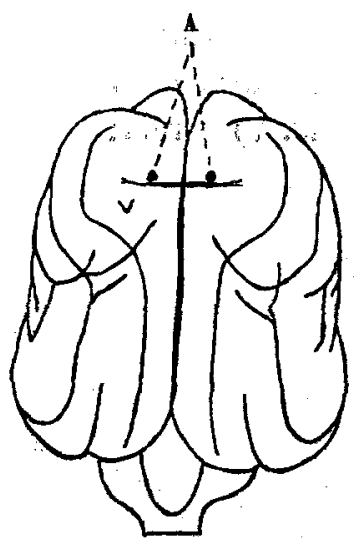

Fig. 9:

wies sich das Gehirn als stark anämisch, was zweifellos von der starken Blutung aus Sinus transvers. der harten Hirnhaut während der Schädeleröffnung abhing. Auf Reizung mittelst des elektrischen Stromes bei einem Rollenabstand von $12 \mathrm{~cm}$ und später von $15-16-17-18 \mathrm{~cm}$ traten ein:

1. Strecken und Abduktion der gekreuzten Vorderextremität mit Spreizung der Zehen bei Reizung des Punktes $A$ (Fig. 9).

Dann war auch bei einem Rollenabstand von $12-10-8 \mathrm{~cm}$ ein motorischer Effekt von keinem Punkte der Hirnrinde aus auszulösen. Bei wiederholten und zahlreichen Reizungen des móntorisêhen Rindengebietes starb das Tier, ohne dass eine Zackung der Extremitäten eingetreten war. 
Zur Frage über die Erregharkeit der mot. Zentra in der Hirnrinde etc. 61

Experiment am 13. Augnst 1908. Nr. 7.

Hund, geboren am 27. August 1908. Zur Zeit des Experimentes sieht er schon mit beiden Augen. Körpergewicht: $840 \mathrm{~g}$; Körperlänge: $34 \mathrm{~cm}$. Bei Eröffnung des Schädels - Blutung aus dem Sinus longitudinalis superior, die zwar bald gestillt werden konnte, immerhin aber den Hund bedentend entkräftete. Reizung der Hirnrinde war bei einem Rollenabstand von $18-15 \mathrm{~cm}$ von keinem motorischen Effekt begleitet; bei einem Rollenabstand von $12 \mathrm{~cm}$ traten ein :

1. Biegen des Rumpfes mit der Konkavität nach der entgegengesetzten Seite bei Reizung des Punktes $R$ (Fig. 10);

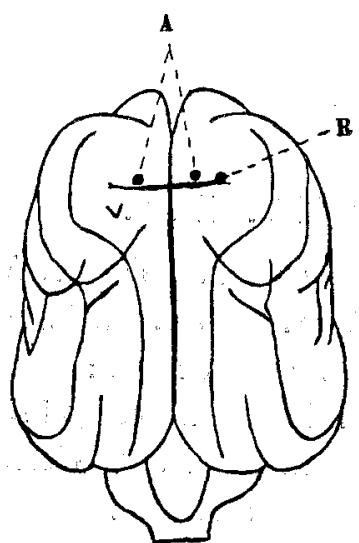

Fig. 10.

2. Aufheben der gekreuzten Vorderpfote im Carpo-metacarpalGelenk auf Reizung des Gebietes am den Punkt $A$ (Fig. 10) herum;

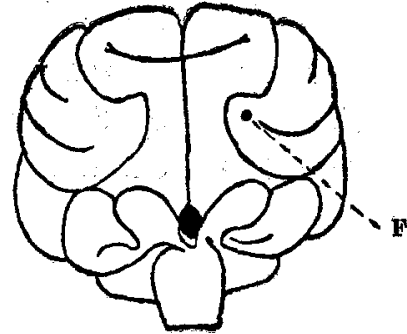

Fig. 11.

3. Bewegung des Kopfes um die senkrechte Achse (d. h. Seitwärtsbewegung des Kopfes) nach der entgegengesetzten Seite auf Reizung des Punktes $F$ (Fig. 11).

Beim Ineinanderschieben der Rollen gelang es nicht, einen epileptischen Anfall hervorzurufen.

\section{Experiment am 17. August 1908. Nr. 8.}

Junger Hund, geboren in der Nacht vom 16. auf den 17. August 1908. Zur Zeit des Experimentes war er 15 Stunden alt. Körpergewicht: $410 \mathrm{~g}$; Körperlänge: $15 \mathrm{~cm}$. Reizung des motorischen Rindengebietes löste bei eiuem Rollenabstand von $11 \mathrm{~cm}$ aus:

1. Kontraktion der gekreuzten Vorderextremität auf Reizung des Punktes $A$ (Fig. 1); 
2. Anziehen der gekreuzten Hinterextremität auf Reizung des Punktes $B$ (Fig. 1);

3. Eine dem Kauakt ähnliche Bewegung der Kiefer auf Reizung des Punktes $C$ (Fig. 1);

4. Bewegung des Kopfes um die senkrechte Achse nach der entgegengesetzten Seite bei Reizung des Punktes $F$ (Fig. 12).

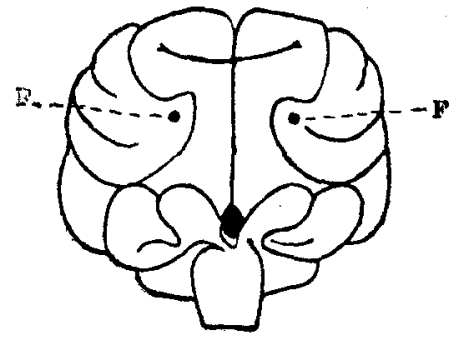

Fig. 12.

Experiment am 23. August 1908. Nr. 9.

Junger Hund, geboren in der Nacht vom 16. auf den 17. August 1908. Körpergewicht: $600 \mathrm{~g}$; Körperlänge: $28 \mathrm{~cm}$. Reizung der Hirnrinde löste bei einem Rollenabstand von $15 \mathrm{~cm}$ aus:

1. Bewegung der gekreuzten

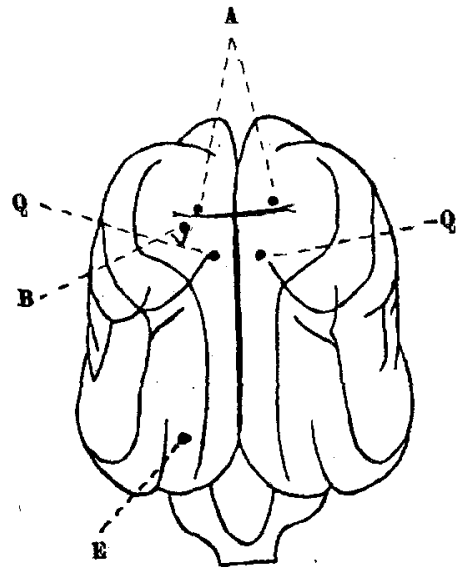

Fig. 13. Vorderextremität auf Reizung des Punktes $A$ (Fig. 13).

2. Konjugierte Bewegung der Augen auf Reizung des Punktes $\boldsymbol{E}$

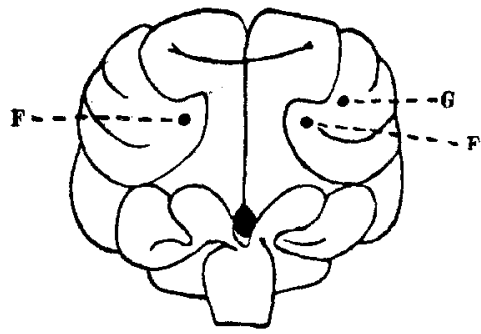

Fig. 14 .

(Fig. 13) an der linken Hemisphäre, wobei sich das rechte Ange nach unten aussen, das linke nach unten innen bewegte;

3. Bewegung des Schwanzes, vornehmlich ein Einkneifen desselben, bei Reizung des Punktes $Q$ (Fig. 13); 
Zur Frage über die Erregbarkeit der mot. Zentra in der Hirnrinde etc. 63

4. Anziehen und Aufheben der gekreuzten Hinterextremität bei Reizung des Punktes $B$ (Fig. 13);

5. Bewegung des Kopfes um die sagittale Körperachse, wobei sich das Frontalgebiet nach der Seite der Reizung, das Occipitalgebiet in entgegengesetzter Richtung bewegt, bei Reizung des Punktes $\boldsymbol{G}$ (Fig. 14);

6. Bewegung des Kopfes nach der gereizten Hemisphäre entgegengesetzten Seite bei Reizung des Punktes $F$ (Fig. 14).

Bei einem Rollenabstand von $5-3-2 \mathrm{~cm}$ und bei einem vollständigen Ineinanderschieben desselben wurden Krämpfe nicht beobachtet.

\section{Experiment am 25. August 1908. Nr. 10.}

Junger Hund, geboren in der Nacht vom 16. auf den 17. August 1908. Körpergewicht: $680 \mathrm{~g}$; Körperlänge: $31 \mathrm{~cm}$. Reizung der Hirnrinde gab bei einem Rollenabstand von $15 \mathrm{~cm}$ :

1. Aufheben der gekreuzten Vorderextremität bei Reizung des Punktes $A$ (Fig. 15);

2. Anziehen der gekreuzten Hinterextremität bei Reizung des Punktes $B$ (Fig. 15);

3. Verbreiterung der Pupille bei Reizung des Punktes $D$ (Fig. 15);

4. Verbreiterung der Pupille bei Reizung des Punktes $N$ (Fig. 15);

5. Ausstrecken der gekreuzten Hinterextremität bei Reizung des Punktes $H$ (Fig. 15);

6. Bewegung der Augäpfel nach oben und nach der entgegengesetzten Seite bei Reizung des Punktes $E$ (Fig. 15);

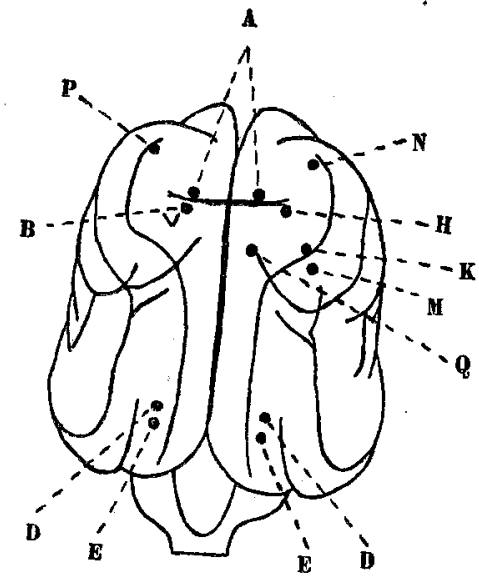

Fig. 15.

7. Bewegung des Schwanzes bei Reizung des Punktes $Q$ (Fig. 15);

8. Kontraktion der Nackenmuskulatur mit Zurückwerfen des Kopfes nach oben und hinten bei Reizung des Punktes $P$ (Fig. 15);

9. Zuckungen in den basalen Teilen der Augenlider, welche bei diesem Experiment entfernt worden waren, bei Reizung des Punktes $K$ (Fig. 15);

10. Bewegung der Oberlippe bei Reizung des Punktes $\boldsymbol{M}$ (Fig. 15). 
Auch bei völligem Ineinanderschiebèn der beiden Spiralen des Induktionsapparates gelang es nicht, Krämpfe hervorzurufen.

Experiment am 27. August 1908. Nr. 11.

Junger Hund, geboren in der Nacht vom 16. auf den 17. August 1908. Körpergewicht: $880 \mathrm{~g}$; Körperlänge: $36 \mathrm{~cm}$. Auf : Reizung mittelst des elektrischen Stromes bei einem Rollenabstand von $15 \mathrm{~cm}$ derselben Punkte der Hirnrinde, wie beim Hund Nr. 10, trat derselbe Effekt ein, mit Ausnahme der Punkte $Q$ und $P$, deren Reizung auch mit stärkereu Strömen $(12-10 \mathrm{~cm})$ ohne Effekt blieb.

Krämpfe gelang es nicht hervorzurufen.

Experiment am 29. Angust 1908. Nr. 12.

Junger Hund, geboren am 28. August 1908. Körpergewicht: $480 \mathrm{~g}$; Körperlänge: $17 \mathrm{~cm}$. Auf Reizung des motorischen Rindengebietes bei einem Rollenabstand von $12 \mathrm{~cm}$ traten ein:

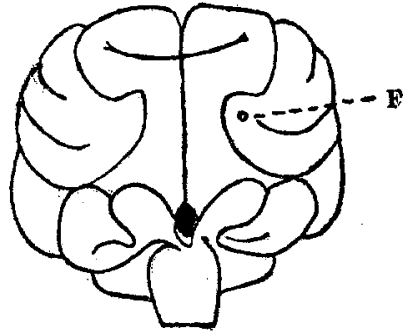

Fig. 16.

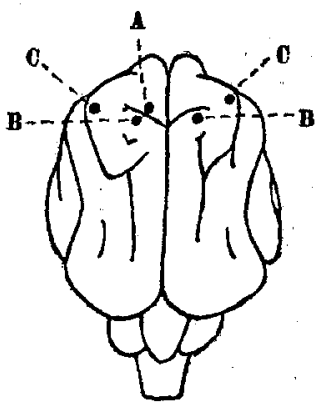

Fig. 17.

1. Kontraktion der gekreuzten Vorderextremität bei Reizung des Punktes $A$ (Fig. 17);

2. Kontraktion der gekreuzten Hinterextremität bei Reizung des Punktes $B$ (Fig. 17);

3. Drehung des Kopfes nach der entgegengesetzten Seite um die vertikale Achse bei Reizung des Punktes $F$ (Fig. 16).

Experiment am 30. August 1908. Nr. 13.

Junger Hund, geboren am 28. August 1908. Körpergewicht: $520 \mathrm{~g}$; Körperlänge: $20 \mathrm{~cm}$. Bei Eröffnung des Schädels wurde der Occipitallappen der linken Hemisphäre leicht beschädigt. Reizung des motorischen Rindengebietes gab: 
Zur Frage über die Erregbarkeit der mot. Zentra in der Hirnrinde etc. 65

1. Anziehen der gekreuzten Vorderextremität bei Reizung des Punktes $A$ (Fig. 17);

2. dem Kauakt ähnliche Kieferbewegungen bei Reizung des Punktes $C$ (Fig. 17).

\section{Experiment am 1. September 1908. Nr. 14.}

Junger Hund, geboren am 28. August 1908. Körpergewicht: $570 \mathrm{~g}$; Körperlänge: $23 \mathrm{~cm}$. Reizung des motorischen Rindengebietes bei einem Rollenabstand von $14 \mathrm{~cm}$ rief hervor:

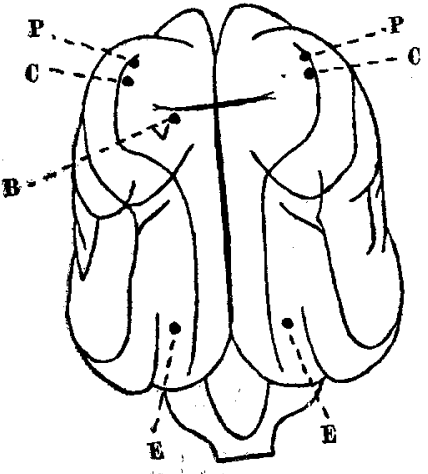

Fig. 18,

1. Bewegung der gekreuzten Hinterextremität bei Reizung des Punktes $B$ (Fig. 18);

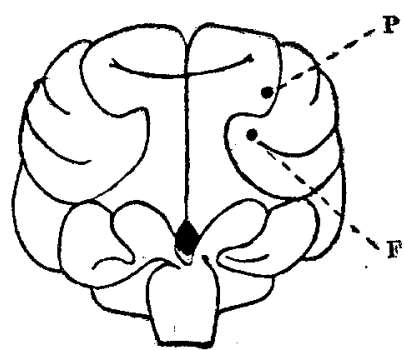

Fig. 19.

2. Kaubewegungen der Kiefer bei Reizung des Punktes $C$ (Fig. 18);

3. Bewegungen der Augen nach der entgegengesetzten Seite und nach unten bei Reizung des Punktes $\boldsymbol{E}$ (Fig. 18);

4. Bewegung des Kopfes nach der entgegengesetzten Seite um die vertikale Achse bei Reizung des Punktes $F$ (Fig. 19);

5. Bewegung des Kopfes nach oben und hinten durch Kontraktion der Nackenmuskulatur bei Reizung des Punktes $P$ (Fig. 18 und 19).

Experiment am 3. September 1908. Nr. 15.

Junger Hund, geboren am 28. August 1908. Körpergewicht: $610 \mathrm{~g}$; Körperlänge: $24 \mathrm{~cm}$. Reizung der Hirnrinde ruft bei einem Rollenabstand von $15 \mathrm{~cm}$ hervor:

1. Auftreten der gekreuzten Vorderextremität bei Reizung des Punktes $A$ (Fig. 20);

2. Anziehen der gekreuzten Hinterextremität bei Reizung des Punktes $B$ (Fig. 20); 
3. konjugierte Bewegung der Augenäpfel nach der entgegengesetzten Seite und nach oben bei Reizung des Punktes $E$ (Fig. 20);

4. Bewegung des Kopfes um die sagittale Körperachse, wobei sich das Frontalgebiet nach der Seite der Reizung bewegt, bei Reizung des Punktes $G$ (Fig. 21);

5. Bewegung des Kopfes um die sagittale Körperachse, wobei ich das Frontalgebiet nach der der Reizung entgegengesetzten Seite

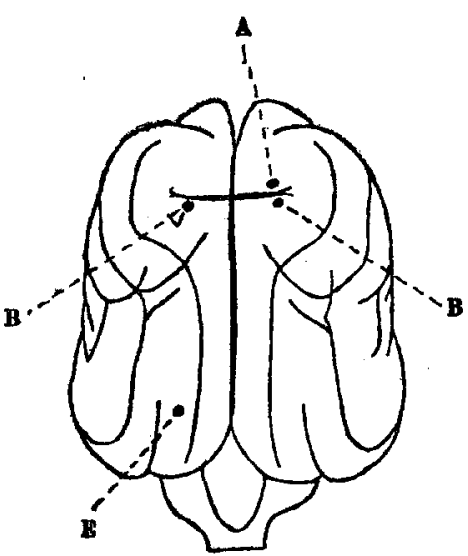

Fig. 20. bewegt, bei Reizung des Punktes $S$ (Fig. 21).

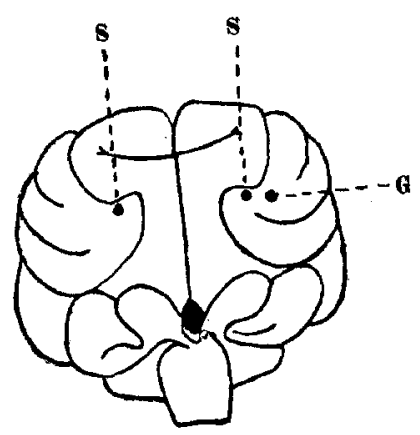

Fig. 21.

Experiment am 11. September 1908. Nr. 16.

Junger Hund, geboren am 28. August 1908. Körpergewicht: $780 \mathrm{~g}$; Körperlänge: $35 \mathrm{~cm}$. Reizung der Hirnrinde bei einem Rollenabstand von $16 \mathrm{~cm}$ gab:

1. Kontraktion der gekreuzten Vorderextremität bei Reizung des Punktes $A$ (Fig. 22);

2. Kontraktion der gekreuzten Hinterextremität bei Reizung des Punktes $B$ (Fig. 22);

3. Kaubewegungen der Kiefer bei Reizung des Punktes $C$ (Fig. 22);

4. Verbreiterung der Pupille bei Reizung des Punktes $D$ (Fig. 22);

5. Bewegung der Augenäpfel bei Reizung des Punktes $E$ (Fig. 22) an der linken Hemisphäre, wobei sich das rechte Auge nach oben aussen, das linke nach oben innen bewegte;

6. Drehung des Kopfes um eine senkrechte Achse nach der entgegengesetzten Seite bei Reizung des Punktes $F$ (Fig. 23);

7. Bewegung des Kopfes um die sagittale Körperachse, wobei sich das Occipitalgebiet nach der der Reizung entgegengesetzten Seite bewegte; bei Reizung des Punktes $G$ (Fig. 23); 
Zur Frage über die Erregbarkeit der mot. Zentra in der Hirnrinde etc. 67

8. Verbreiterung der Pupille bei Reizung des Punktes N (Fig. 22);

9. Kontraktion der Nackenmuskulatur mit Zurückwerfen des Kopfes nach oben und hinten bei Reizung des Punktes $P$ (Fig. 22);

10. Bewegung des Schwanzes bei Reizung des Punktes $Q$ (Fig. 22);

11. Biegen des Rumpfes mit der Konkavität nach der entgegengesetzten Seite bei Reizung des Punktes $R$ (Fig. 22).

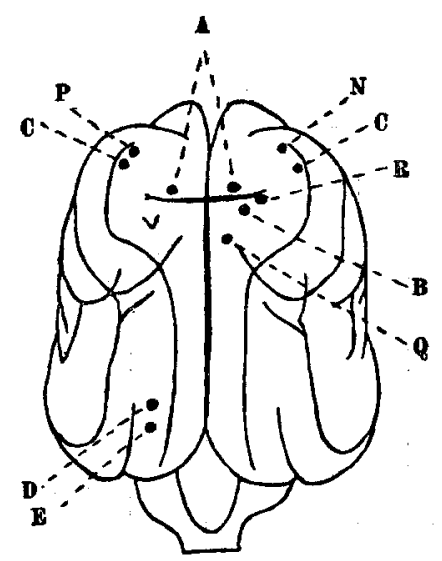

Fig. 22.

In Anbetracht der Bestimmtheit der Ergebnisse, welche durch die eben beschriebenen Experimente erzielt worden sind, ersehien

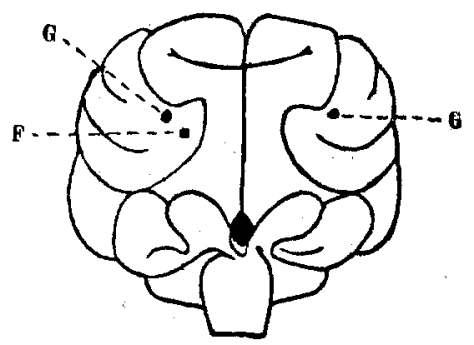

Fig. 23.

es als zulässig, sich auf diese 16 Experimente, welche an jungen Tieren aus vier Würfen angestellt worden waren, zu beschränken. Sie umfassten die Altersstufen von 12 Stunden bis (inklusive) 16 Tage nach der Geburt. Bevor aber irgendwelche Schlüsse aus den angegebenen Tatsachen gezogen werden, wollen wir noch einige an Meerschweinchen angestellte Experimente erwähnen.

An neugeborenen Meerschweinchen hatte ich die Absicht, mich bloss von folgendem zu überzeugen - wovon keiner der früheren Autoren Erwähnung tut - : Ist es möglich, bei diesen Tieren in den ersten Tagen nach der Geburt durch Reizung des motorischen Rindengebietes Krämpfe (klonische sowohl als tonische) hervorzurufen? Die Antwort fiel positiv aus.

Experiment am 17. November 1908. Nr. 17, 18, 19.

Meerschweinchen, geboren am 13. November 1908. Der Schädel wird eröffnet. Reizung des motorischen Rindengebietes ruft unter der Bedingung, dass sie von gewisser Dauer ist (20-30 Sek.) bei einem Rollenabstand von $12-10 \mathrm{~cm}$ die erwähnten Krämpfe hervor. 
Zwei Meerschweinchen, geboren in der Nacht auf den 17 . November 1908, also einige Stunden alt. Der Schädel wird eröffnet. Reizung des motorischen Rindengebietes dieser Tiere ruft unter den gleichen Bedingungen und bei der gleichen Stromstärke die typischen Krämpfe hervor.

Um die Ergebnisse der beschriebenen. Experimente anschaulich zu machen, gruppieren wir sie in Form einer Tabelle, in welcher das Zeichen $(+)$ gegenüber der Bezeichnung des Punktes $(A, B$, $C$... usw.), dessen Reizung einen bestimmten motorischen Effekt beim gegebenen Tiere auslöste, gestellt ist:

\begin{tabular}{|c|c|c|c|c|c|c|c|c|c|c|c|c|c|c|c|c|c|c|}
\hline $\begin{array}{c}\text { Alter } \\
\text { des } \\
\text { Hundes }\end{array}$ & $\begin{array}{c}\text { Zeit des } \\
\text { Experi- } \\
\text { mentes } \\
1908\end{array}$ & $A$ & $B$ & $C$ & $D$ & $E$ & $F$ & $G$ & $H$ & $K$ & $M$ & $N$ & $O$ & $P$ & $Q$ & $R$ & $S$ & $T$ \\
\hline $\begin{array}{rl}12 & \text { Stdn. } \\
15 & \# \\
24 & " \\
2 & \text { Tage } \\
3 & " \\
4 & " \\
5 & " \\
6 & " \\
7 & " \\
7 & " \\
8 & " \\
9 & " \\
10 & " \\
11 & " \\
14 & " \\
16 & "\end{array}$ & $\begin{array}{l}\text { 27. Juni } \\
\text { 17. Aug. } \\
29 . \text { " } \\
\text { 30. "”. } \\
\text { 31. Juli } \\
\text { 1. Sept. } \\
\text { 2. Aug. } \\
\text { 3. Sept. } \\
\text { 4. Aug. } \\
\text { 23. } " \\
\text { 5. Juli } \\
\text { 25. Aug. } \\
\text { 7. Juli } \\
\text { 27. Aug. } \\
\text { 11. Sept. } \\
\text { 13. Aug. }\end{array}$ & $\begin{array}{l}+ \\
+ \\
+ \\
+ \\
+ \\
+ \\
+ \\
+ \\
+ \\
+ \\
+ \\
+ \\
+ \\
+\end{array}$ & $\begin{array}{l}+ \\
+ \\
+ \\
+ \\
+ \\
+ \\
+ \\
+ \\
+ \\
+ \\
+ \\
+ \\
+\end{array}$ & $\begin{array}{l}+ \\
+ \\
+\end{array}$ & $\begin{array}{l}+ \\
+ \\
+ \\
+\end{array}$ & $\begin{array}{l}+ \\
+ \\
+ \\
+ \\
+ \\
+\end{array}$ & $\begin{array}{l}+ \\
+ \\
+ \\
+ \\
+\end{array}$ & + & $\begin{array}{l}+ \\
+ \\
+\end{array}$ & $\begin{array}{l}+ \\
+ \\
+\end{array}$ & $\begin{array}{l}+ \\
+ \\
+\end{array}$ & $\begin{array}{l}+ \\
+ \\
+ \\
+ \\
+\end{array}$ & + & $\begin{array}{l}+ \\
+ \\
+ \\
+\end{array}$ & $\begin{array}{l}+ \\
+ \\
+ \\
+ \\
+ \\
+\end{array}$ & $\begin{array}{l}+ \\
+ \\
+\end{array}$ & + & + \\
\hline
\end{tabular}

Aus den angeführten Untersuchungen folgt also:

1. dass die Hirnrinde neugeborener Hunde schon in den ersten $S$ tunden nach der Geburt durch den elektrischen Strom erregbar ist, wenn nur bei Ausführung der Operation alle nötigen Massregeln getroffen worden sind; wobei nur eine geringe Anzahl motorischer Zentren erregbar erscheint;

2. dass die nötige Stromstärke mit dem zunehmenden Alter des Tieres abnimmt, wobei in den nächstfolgenden Tagen die Zentren sich weiterentwickeln und ihre Zahl sich folglich vermehrt;

3. dass sich unter dem Einfluss der genanten Reizung die Zentren neugeborener Hunde ausserordentlich schnell erschöpfen; 
Zur Frage über die Erregbarkeit der mot. Zentra in der Hirnrinde etc. 69

4. dass es während der ersten 24 Stunden auszulösen gelingt: allgemeine Kontraktion der gekreuzten Vorderextremität bei Reizung des Punktes $A$; allgemeine Kontraktion der gekreuzten Hinterextremität bei Reizung des Punktes $B$; dem Kaukt ähnliche Bewegungen der Kiefer bei Reizung des Punktes $C$; Drehung des Kopfes um eine senkrechte Achse nach der entgegengesetzten Seite bei Reizung des Punktes $F$; Kontraktion der Nackenmuskulatur mit Zurückwerfen des Kopfes nach oben und hinten bei Reizung des Punktes $P$;

5. dass bei einem 3 Tage alten Hunde ausser den genannten noch der Punkt $E$ erregbar ist, bei dessen Reizung eine Seitwärtsbewegung der Augen, welche sich mit einer Bewegung bald nach oben, bald nach unten kombiniert, eintritt;

6. dass bei einem 5 Tage alten Hunde ausser den angeführten noch folgende Bewegungen auslösbar sind: Drehung des Kopfes um die sagittale Körperachse, wobei sich das Occipitalgebiet nach der der Reizung entgegengesetzten Seite bewegt, bei Reizung des Punktes $G$; eine ähnliche Bewegung abernurnach der entgegengesetzten Seite bei Reizung des Punktes $S$; Schliessen der Augenlider bei Reizung des Punktes $K$; Aufrichten des Ohres bei Reizung des Punktes $T$;

7. dass es bei einem 7 Tage alten Hunde gelingt, eine Bewegung des Schwanzes bei Reizung des Punktes $Q$ auszulösen, ausser den übrigen von den entsprechenden Punkten auslösbaren Bewegungen;

8. dass bei einem 9 Tage alten Hunde ausser den genanten Bewegungen beobachtet werden kann: Verbreiterung der Pupille bei Reizung der Punkte $D$ und $N$; Bewegung der Oberlippe bei Reizung des Punktes $M$; Ausstrecken der gekreuzten Hinterextremität bei Reizung des Punktes $H$;

9. dass es bei einem 10 Tage alten Hunde ausser den genanten Bewegungen noch hervorzurufen gelinkt: konjugierte Bewegungen der Augäpfel nach der entgegengesetzten Seite, wobei siestets mit einer 
gleichzeitigen Bewegung nach oben oder unten kombiniert war, bei Reizung des Punktes $O$; Biegendes Rumpfes mit derKonkavität nach der entgegengesetzten Seite bei Reizung des Punktes $R$;

10. dass sich folglich mit dem zunehmenden Alter der neugeborenen Hunde die Zahl der erregbaren Punkte an der Rindenoberfläche vergrössert;

11. dass einzelne der erwähnten Punktenicht bei allen Tieren der gleichen Art und des gleichen Alters erregbar erscheinen;

12. dass der Unterschied des neugeborenen vom erwachsenen Tiere in bezug auf die motorischen Zentren, abgesehen von der geringeren Anzahl derselben, hauptsächlich darin besteht, dasses a) bei neugeborenen Hunden nicht gelingt, klonische und tonische Krämpfe auszulösen, und dass b) bei Reizung einzelner Punte ein allgemeiner summariseher motorischer Effekt auftritt, während beim erwachsenen Tiere unter den gleichen Bedingungen mehr partielle, differenzierte und abgegrenzte Bewegungen hervorgerufen werden. Das betrifft z. B. die allgemeine Kontraktion der Extremitat des Nengeborenen und die Bewegung in den einzelnen Gelenken beim erwachsenen Tiere, ebenso die Augenbewegungen [siehe Gerwer ${ }^{1}$ )]

13. dass bei Reizung des motorischen Rindengebietes neugeborener Meerschweinchen, die einige Stunden vor dem Experiment zur Welt kamen, ein klares Bild klonischer, später in tonische übergehender Krämpfe auftritt.

Das für die Fig. 1, 12, 16, 17 benutzte Gehirnschema stammt von einem 15 Stunden alten Hündehen, das für die übrigen Figuren benutzte von einem 6 Tage alten.

1) Gerwer, Über die Gehirnzentren für die Bewegung der Augen. Dissert. St. Petersburg 1899. Russisch. 